\title{
Hypergammaglobulinemic purpura of waldenstorm associated with sjogren's syndrome in a young female responding to rituximab treatment
}

\author{
Neeraj Jain*, Lalit Duggal \\ Department of Rheumatology \& Clinical Immunology, Sir Ganga Ram Hospital, New Delhi, India; \\ *Corresponding Author: neerajamita@yahoo.com
}

Received 27 January 2013; revised 1 April 2013; accepted 15 April 2013

Copyright (C) 2013 Neeraj Jain, Lalit Duggal. This is an open access article distributed under the Creative Commons Attribution License, which permits unrestricted use, distribution, and reproduction in any medium, provided the original work is properly cited.

\begin{abstract}
Hypergammaglobulinemic Purpura of Waldenstorm is one of the uncommon conditions with purpura and is often associated with collagen vascular disease. It is difficult to treat and sometimes needs anti CD 20 molecules for resistant cases.
\end{abstract}

Keywords: Hypergammaglobulinemic Purpura of Waldenstorm; Sjogren's Syndrome; Rituximab

\section{CASE HISTORY}

We present a case of 20 years old female who came to us in June 2008 with recurrent history of rash since last 1 year over both legs, rash was precipitated on prolonged standing and disappeared with rest in 2 - 3 days and was associated with pain she also complained of dryness of mouth and dryness of eyes and received steroids for last 1 year but showed no improvement. There was no history of fever, joint pains, photosensitivity. On admission she was conscious, oriented afebrile, pulse was $76 / \mathrm{min}$, BP 120/76 mmHg. Systemic examination was normal. She had maculopapular rash over lower limbs which was non blanch able. Investigations showed Hemoglobin 9.9 mg\%, TLC 3800/cumm, ESR $98 \mathrm{~mm}$ in 1st hour, CRP was positive, Renal and Liver function were within normal limits. Further skin biopsy showed leucocytoclastic vasculitis, in view of biopsy report CT scan was done to look for primary cause it revealed mediastinal lymphadenopathy, endoscopic ultrasound FNAC done revealed granulomatous changes with focal necrosis, montoux test was $25 \mathrm{~mm}$ and TB quantiferon gold test was positive. So patient was diagnosed as having tubercular mediastinal lymphadenopathy with leucocytoclastic vasculitis and was started on 4 drugs antitubercular drugs. She was subsequentely followed up as outpatient, after 8 weeks she came with similar complaints so she underwent further test. Her Rheumatoid factor was positive. ANA was positive in fine speckled pattern, ENA profile by BINDAZYME IMMUNOASSAY showed high titre of SSA/RO, SSB/La [1] and Sm/RNP autoantibody. Her immunoglobulin profile showed highly raised IgG level 2831 (650 - 1600) mg/dL and normal IgA IgM level. Hepatitis B and C were negative. So a final diagnosis of Hypergammaglobulinemic Purpura of Waldenstorm associated with Sjogren's Syndrome with leucocytoclastic vasculitis was made and she was started on hydroxychloroquine 200 twice daily. She initially responded but again had flare of rashes so after she finished her tubercular treatment was put on azathioprine. She was seen periodically but she persisted to have same complaints even 1year after she was put on disease modifying drugs, so she was finally treated with rituximab two doses in January 2010. She responded to rituximab and after 2 year she is symptom free and is on hydroxychloroquin plus supportive medications.

\section{DISCUSSION}

Hypergammaglobulinemic purpura of Waldenström is characterized by hypergammaglobulinemia, recurring purpura, an elevated erythrocyte sedimentation rate, and the presence of rheumatoid factor indicative of circulating immune complexes. There is an association with autoimmune diseases, especially Sjögren's syndrome [2] and lupus erythematosus. Hypergammaglobulinemic purpura is considered primary when there is no other associated disease or secondary when associated with other diseases, usually autoimmune. Immune derangements are fundamental in its pathogenesis, although its cause is still unknown [3]. Therapy is generally unnecessary as this is usually benign condition, practically treatment with hy- 
droxychloroquine is enough and if required azathioprine may be added. Rituximab seems to be safe and effective in the treatment of patients with a variety of autoimmune diseases that are refractory to other modalities of treatment [4].

\section{REFERENCES}

[1] Capra, J.D. (1971) Clinical and immunologic observations in hypergammaglobulinemic purpura. Mount Sinai Journal of Medicine, 38, 375.
[2] Ferreiro, J.E., Pasarin, G., et al. (1986) Benign hypergammaglobulinemic purpura of Waldenstrom associated with Sjogren's syndrome. American Journal of Medicine, 81, 734-740. doi:10.1016/0002-9343(86)90569-3

[3] Finder, K.A., et al. (1990) Hypergammaglobulinemic purpura of Waldenström. Journal of the American Academy of Dermatology, 23, 669-676. doi:10.1016/0190-9622(90)70271-I

[4] Scheinberg, M., et al. (2006) Rituximab in refractory autoimmune diseases: Brazilian experience with 29 patients (2002-2004). Clinical and Experimental Rheumatology, 24, 65-69. 